\title{
Trastornos de la conducta alimentaria como factor de riesgo para osteoporosis
}

\author{
Ma Teresa Rivera-Gallardo, LN, (1) Ma del Socorro Parra-Cabrera, Dr en C, ${ }^{(2)}$ \\ Jorge Armando Barriguete-Meléndez, Psiq. ${ }^{(3)}$
}

\author{
Rivera-Gallardo MT, Parra-Cabrera MS \\ Barriguete-Meléndez JA. \\ Trastornos de la conducta alimentaria \\ como factor de riesgo para osteoporosis. \\ Salud Publica Mex 2005;47:308-318.
}

\section{Resumen}

Los trastornos de la conducta alimentaria son comunes en mujeres jóvenes con una prevalencia estimada de entre 4-5\%. La pérdida de masa ósea es una complicación física de la anorexia nervosa y trastorno alimentario no especificado que afecta tanto a hueso cortical como trabecular. El efecto sinérgico de la desnutrición y la deficiencia de estrógenos produce una pérdida de masa ósea a través del desacoplamiento entre resorción osteoclástica y formación osteoblástica. La severidad varía dependiendo de la duración de la enfermedad, el peso menor alcanzado y la actividad física. La repercusión a largo plazo es evidente pues existe un incremento en el riesgo de fractura en las pacientes que han padecido anorexia nervosa. La primera línea de tratamiento para recuperar la masa ósea es la rehabilitación nutricia y un incremento de peso. La terapia de reemplazo hormonal podría ser efectiva si se combina con métodos anabólicos. Los términos osteopenia y osteoporosis fueron adoptados para definir la deficiencia de masa ósea en adultos. Los autores de las publicaciones que fueron revisadas utilizaron dichos términos para definir datos densitométricos en sujetos jóvenes que no han alcanzado la masa ósea pico. Sugerimos el término "hipo-osteogenesia" para definir el desarrollo deficiente de masa ósea en adolescentes o niños.

Palabras clave: pérdida de masa ósea; anorexia nervosa; trastorno alimentario no especificado; terapia sustitutiva hormonal; hipo-osteogenesia

\author{
Rivera-Gallardo MT, Parra-Cabrera MS, \\ Barriguete-Meléndez JA. \\ Eating disorders \\ as risk factors for osteoporosis. \\ Salud Publica Mex 2005;47:308-318.
}

\section{Abstract}

Eating disorders (TCA per its abbreviation in Spanish) are common in young women, with an estimated prevalence of $4-5 \%$. One of the physical complications of eating disorders, especially anorexia nervosa (AN) and eating disorder not otherwise specified (TANE) is bone mass loss, which affects both cortical and trabecular bone. The synergistic effect of malnutrition and estrogen deficiency produces significant bone mass loss, resulting from the uncoupling of bone turnover characterized by a decrease in osteoblastic bone formation and an increase in osteclastic bone resorption. The mechanisms implied in the pathogenesis of bone loss are the hypoestrogenism, hypercortisolism, serum leptin levels and insulin-like growth factor decrease. Severity of bone loss in anorexia nervosa varies depending on duration of illness, the minimal weight ever and sedentarism or strenuous exercise. Long term consequences occur, such as a fracture risk increase in patients who have suffered anorexia nervosa, compared with the general population. The first treatment line to recover bone mass is nutritional rehabilitation together with weight gain. Hormonal replacement therapy may be effective if combined with an anabolic method. Osteopenia and osteoporosis are terms adopted to define the deficiency of bone mass in adults. Authors have used these terms to define densitometric data in young subjects who have not reached their peak bone mass. We suggest the term "hypo-osteogenesia" to define the deficiency in the development of bone mass in adolescents or children.

Key words: bone mass loss; anorexia nervosa; eating disorder not otherwise specified; hormonal substitutive therapy; hipo-osteogenesia

(I) Grupo Médico Río Mayo, Fundación Ariwá para el Tratamiento, Prevención e Investigación de los Trastornos de la Conducta Alimentaria. Cuernavaca, Morelos, México.

(2) Centro de Investigación en Nutrición y Salud, Instituto Nacional de Salud Pública. Cuernavaca, Morelos, México.

(3) Fundación Franco Mexicana para la Medicina, Departamento de Neurología y Psiquiatría, Instituto Nacional de Ciencias Médicas y Nutrición Salvador Zubirán. México, DF.

Fecha de recibido: 30 de noviembre de 2004 - Fecha de aprobado: 8 de julio de 2005

Solicitud de sobretiros: Ma Teresa Rivera Gallardo. Avenida Río Mayo I306 interior 10I, colonia Vista Hermosa 62290. Cuernavaca, Morelos, México. Correo electrónico: terenut@prodigy.net.mx 
$\mathbf{L}$ os trastornos de la conducta alimentaria (TCA) son síndromes psiquiátricos ${ }^{1,2}$ en los cuales la anorexia nervosa (AN) se caracteriza por un miedo intenso a subir de peso, rechazo a mantener el peso mínimo esperado para la talla y edad, amenorrea y alteración en la percepción de la imagen corporal. Puede o no haber episodios de "atracón" seguidos del uso de purgas o métodos compensatorios. ${ }^{1}$ La bulimia nervosa (BN) presenta episodios de atracón y purgas, ejercicio extenuante o ayunos, y el trastorno alimentario no especificado (TANE) es aplicable a aquellas pacientes que aún no reúnen todos los criterios diagnósticos para $\mathrm{AN}$ o BN. ${ }^{1}$ A pesar de que esta clasificación está sujeta a críticas por sus definiciones tan estrechas, ${ }^{3}$ y que los síntomas se presentan a través de un continuo entre los propios de la AN y la $\mathrm{BN}^{4,5}$ facilita a los profesionales clínicos hacer el diagnóstico de un TCA.

La estimación de la incidencia o prevalencia de los TCA varía dependiendo del tamaño y edad de la muestra y de los métodos de evaluación. ${ }^{4,6}$ Estudios en Estados Unidos de América y Europa Occidental encontraron una tasa de prevalencia promedio de $0.3 \%$ para $\mathrm{AN}$, de $1 \%$ para $\mathrm{BN}$ en mujeres jóvenes y $0.1 \%$ para $\mathrm{BN}$ en hombres jóvenes. ${ }^{7}$ En adolescentes españolas se ha reportado una prevalencia que fluctúa entre 0.3 y $0.45 \%$ para $\mathrm{AN}, 0.41$ y $0.8 \%$ para $\mathrm{BN}, 3.1$ y $4.71 \%$ para TANE; entre 4.1 y $5.17 \%$ de la población total sufre un TCA. ${ }^{6,8}$ Los TCA se presentan con una razón de prevalencia hombre/mujer de entre 1:6 y 1:10 ${ }^{4,8}$ Respecto de la incidencia de AN, la tasa global es de por lo menos 8 por 100000 años persona ${ }^{7,9}$ y de BN 12 por 100000 años persona., ${ }^{710}$ Para el grupo de mujeres de 15 a 24 años de edad la incidencia se ha incrementado el último siglo desde $1970 .{ }^{7,9}$ En México no hay datos sobre la incidencia y prevalencia de los TCA en adolescentes pero se ha detectado que $0.9 \%$ de los hombres y $2.8 \%$ de las mujeres en edad estudiantil (12 a 19 años), presentan alto riesgo de padecer un TCA; ${ }^{11}$ la encuesta nacional de epidemiología psiquiátrica en México, en población mayor de 18 años, destacó una prevalencia de bulimia de $0.6 \%$ en hombres y $1.8 \%$ en mujeres. ${ }^{12}$

La AN y BN están relacionadas con una tasa de morbilidad de 33\% después de casi 12 años de la primera admisión en servicios hospitalarios; se han reportado tasas de mortalidad estandarizada de 9.6, siendo las principales causas de muerte las arritmias y enfermedades infecciosas. ${ }^{13}$

El Consenso Mexicano de Osteoporosis de 2000 determina que "la osteoporosis es un trastorno esquelético generalizado caracterizado por una masa ósea disminuida y deterioro de la calidad del tejido óseo, con un aumento subsecuente en la fragilidad del hue- so y mayor riesgo de fractura". ${ }^{14}$ Los criterios diagnósticos fueron propuestos por la Organización Mundial de la Salud para evaluar el riesgo de fractura de cadera en mujeres blancas caucásicas empleando la medición de masa ósea con absorciometría dual de rayos- $X{ }^{15}$ Sin embargo, estos criterios no deben emplearse para evaluar el riesgo de fractura en niños o adolescentes que todavía no alcanzan su masa ósea "pico", es decir, la densidad ósea máxima que se alcanza alrededor de los 20 y 30 años de edad. ${ }^{14}$

Un mecanismo efectivo para reducir el riesgo de padecer osteoporosis en la edad adulta es alcanzar una masa ósea "pico" máxima durante la juventud. ${ }^{16,17} \mathrm{Al}-$ rededor de la mitad del calcio óseo del adulto es depositado durante la adolescencia;, ${ }^{18}$ por ello, la falla en la obtención de suficiente densidad ósea en los años de máxima formación aumenta el riesgo de osteoporosis y fracturas en la edad adulta. ${ }^{19-21}$ Durante esta ventana de oportunidad para alcanzar una mineralización ósea óptima, un alto porcentaje de adolescentes mexicanas podría presentar $\mathrm{TCA}^{11}$ lo que limitaría su desarrollo por diferentes vías ${ }^{21}$ y repercutiría años más tarde en su salud ósea, como se muestra en la figura 1. Los TCA pueden generar múltiples complicaciones que derivan de la desnutrición, así como del uso de purgas, lo que afecta el sistemas cardiovascular, renal, gastrointestinal, endocrino, metabólico y, con mayor frecuencia, el esquelético. ${ }^{22}$ Por ello, en relación con el tema de TCA y su efecto en la densidad mineral ósea (DMO), los autores presentarán los hallazgos más importantes publicados hasta el momento.

El objetivo de este estudio fue realizar una revisión crítica para identificar el efecto de los TCA en la densidad mineral ósea a corto y largo plazo, describir los mecanismos endocrinos y metabólicos involucrados, y las estrategias que hasta el momento se han planteado para su tratamiento.

\section{Material y métodos}

Se revisó la literatura internacional para un periodo de 20 años (1984 - 2004) con el objetivo de obtener artículos que informaran sobre TCA y osteoporosis; para esto se utilizaron medios digitales (Medline) y el servicio mensual de Biomail bajo los siguientes títulos: Osteoporosis AND eating disorders, estrogen replacement therapy in AN, hormone therapy in AN y IGF-1 Therapy in AN. Se identificaron 153 artículos y luego se seleccionaron 102 por estar directamente relacionados con el tema. Los 51 restantes se descartaron por su relación con amenorrea hipotalámica en atletas, y terapia de remplazo hormonal en la perimenopausia. 


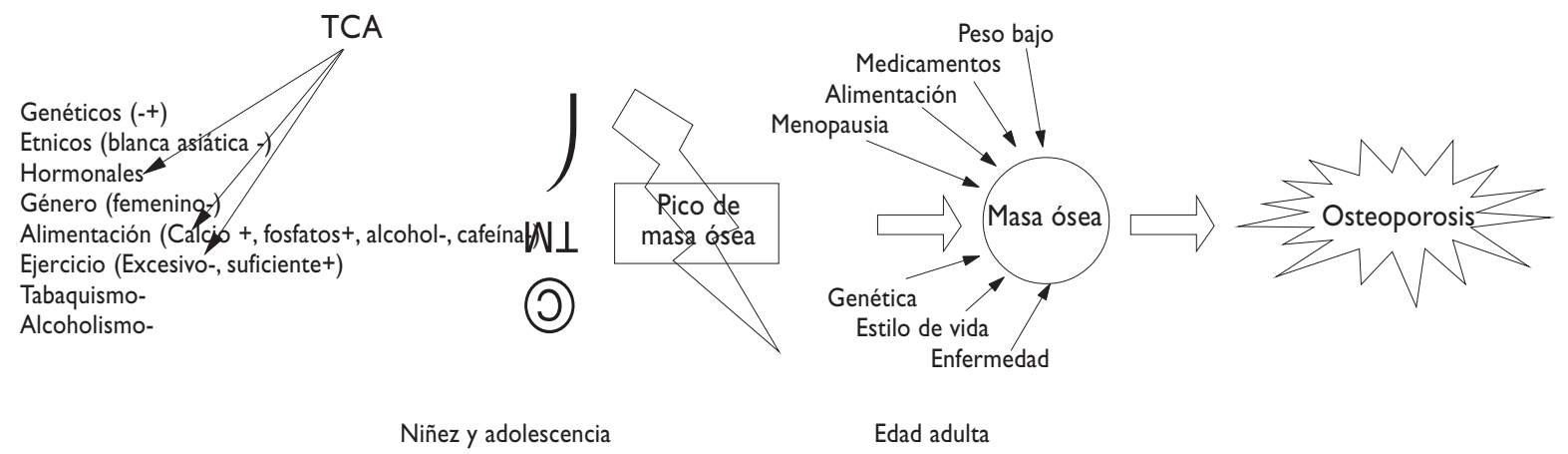

Figura I. Factores Que influyen en el desarrollo de la masa ósea

\section{Resultados}

\section{Trastornos de la conducta alimentaria asociados con osteoporosis}

La pérdida de DMO es una complicación potencialmente crónica de la $\mathrm{AN}^{23-37}$ y TANE, ${ }^{36}$ que en ocasiones ha sido suficiente para ocasionar fracturas patológicas ante trauma mínimo, ${ }^{34,38-43}$ así como un retraso en el crecimiento tanto en mujeres ${ }^{44-46}$ como en hombres adolescentes. ${ }^{47}$ Se ha llegado a estimar una pérdida de estatura de $3 \mathrm{~cm}$ en relación con la esperada en mujeres adolescentes con $\mathrm{AN} .{ }^{48}$ Esto por la alteración en la función de la hormona de crecimiento y niveles bajos del factor de crecimiento parecido a la insulina (FCI-1). ${ }^{49,50}$

Se ha comprobado una DMO ósea disminuida en columna lumbar, ${ }^{24-36,51-58}$ cuello femoral, ${ }^{25-27,36,52,53,56}$ radio distal, ${ }^{37,56}$ calcáneo, cuerpo entero ${ }^{29}$ y cadera total ${ }^{51,52,54}$ en mujeres; y en columna lumbar, cuello femoral ${ }^{59-61}$ y cuerpo entero en hombres con AN. ${ }^{61}$ Por su mayor actividad metabólica, la pérdida ocurre en hueso trabecular antes que en cortical, ${ }^{25,62,63}$ lo que registra pérdidas anuales de masa ósea lumbar de entre 2 y 10\%. . $^{31,64-66}$ Diversos estudios han encontrado que la utilización de purga en la AN no incrementa la pérdida de DMO. ${ }^{28,32,37,51,67}$ Karlsson y su equipo ${ }^{68}$ señalan que el desconocimiento del efecto del tamaño de los huesos en las expresiones de densidad o masa ósea puede ocasionar inferencias erróneas acerca de la patogénesis de la fragilidad ósea.

Respecto de BN, únicamente cuando hay antecedentes de anorexia nervosa presentan DMO disminuida. ${ }^{31,32,69-71}$ En algunos hombres con BN se ha detectado una DMO disminuida en columna lumbar. ${ }^{60}$
En lo relativo a TANE se ha encontrado una disminución de DMO en columna lumbar, radio distal y fémur, ${ }^{36}$ con un incremento en el riesgo de fractura durante varios años después del diagnóstico del TANE. ${ }^{72}$

\section{Trastornos endocrinos y metabólicos que influyen en la sintomatología de los TCA}

La mayoría de los trastornos endocrinos y metabólicos en los TCA son secundarios al estado de inanición y pérdida de peso, a la conducta purgativa o al estrés psicológico. ${ }^{73}$ Existen alteraciones en el eje hipotálamopituitaria-ovarios, adrenal y tiroideo así como en la hormona de crecimiento, insulina, neuropéptidos, opioides, leptina y neurotransmisores. ${ }^{73}$ Estas anormalidades desaparecen con la realimentación y ganancia de peso. ${ }^{74}$ La hormona liberadora de corticotropina, algunos neuropéptidos, la dopamina y opioides endógenos se han propuesto como factores perpetuantes de la conducta anoréctica; en consecuencia, la desnutrición puede contribuir al círculo vicioso sosteniendo y perpetuando el deseo para adelgazar más y hacer dieta. $^{74}$

\section{Mecanismos que desencadenan la deficiencia de la DMO en la AN}

El efecto sinérgico de la desnutrición y la deficiencia de estrógenos produce una pérdida singnificativa de hue$\mathrm{so}^{75,76}$ a través de un desacoplamiento entre resorción osteoclástica y formación osteoblástica, ${ }^{28,29,53-55,58,77-80}$ específicamente cuando el IMC está por debajo de 16.4 $\mathrm{kg} / \mathrm{cm}^{2}{ }^{67}$ Una pérdida generalizada de colágeno seguida del hipoestrogenismo es el factor etiológico más importante para la pérdida de masa ósea en la $\mathrm{AN} .{ }^{24} \mathrm{El}$ 
FCI-1 es una potente hormona osteotrópica, ${ }^{81} \mathrm{y}$ un indicador del estado nutricio en pacientes con $\mathrm{TCA}^{50,82}$ se encuentra disminuido en mujeres con $\mathrm{AN}^{2},{ }^{28,50,57,79,82-84} \mathrm{y}$ su ausencia en las células del hueso ejerce un efecto dañino en la función del osteoblasto. ${ }^{37}$ Los niveles séricos del FCI-1 aumentan con la ganancia de peso $0^{85,86}$ y con la realimentación, ${ }^{79,82,87,88}$ prediciendo la recuperación de la masa ósea en estudios longitudinales. ${ }^{85,86}$ La deficiencia de estrógenos puede ser responsable del déficit en la DMO volumétrica $\left(\mathrm{g} / \mathrm{cm}^{3}\right)$ tanto en columna lumbar y cuello femoral, mientras que la desnutrición puede ocasionar un déficit en el ancho del cuello femoral y cuerpo vertebral. ${ }^{68}$

La leptina ${ }^{88,89}$ puede actuar como factor protector inhibiendo la resorción..$^{90-93}$ La disminución dramática en los niveles de leptina que se observan en la $\mathrm{AN}^{84,88}$ pueden generar la pérdida de hueso a través de una disminución en las tasas de formación de hueso cortical. ${ }^{89}$ Además, la correlación entre la leptina y los marcadores de resorción ósea en pacientes con AN durante el proceso de realimentación, sugiere una relación de causa-efecto entre concentraciones de leptina y el proceso de formación ósea. ${ }^{79}$

Los niveles bajos de triyodotironina en algunas pacientes también pueden estar involucrados en la pérdida de masa ósea. Esto debido a que las hormonas tiroideas tienen efectos sobre el cartílago del crecimiento estimulando la mineralización de la matriz, así como la formación y resorción óseas. ${ }^{90}$

El hipercorticismo también tiene influencia. ${ }^{94}$ Aunque se observa únicamente en una minoría de casos con osteopenia severa, ${ }^{57,95}$ y esto es un indicio de que la pérdida de $\mathrm{DMO}$ no es ocasionada por este mecanismo en la mayoría de las pacientes, quienes lo presentan tienen un mayor déficit en la formación ósea ${ }^{83}$ y pérdida severa de $\mathrm{DMO}$ vertebral. ${ }^{28,33}$ Los niveles de hormona paratiroidea se encuentran normales, por ello se descarta la posibilidad de que el hipercorticismo contribuya al deterioro de la masa ósea vía hiperparatiroidismo secundario. ${ }^{28}$ La ganancia de peso produce una disminución en los niveles de cortisol sérico y un aumento subsecuente en marcadores de formación ósea relacionado directamente con el incremento en FCI-1. ${ }^{83}$

En cuanto a los factores dietéticos, la ingestión de calcio y vitamina $\mathrm{D}$ en mujeres con $\mathrm{AN}$ puede ser muy baja $\mathrm{a}^{29,37,62}$ ya que los lácteos se perciben como alimentos grasos. ${ }^{20,62}$ Además, el hipoestrogenismo puede generar un aumento en el requerimiento de calcio ${ }^{56} \mathrm{y}$ la acidosis metabólica, una consecuencia del deterioro nutricio, estimula directamente la liberación de calcio del tejido óseo. Si ésta resulta prolongada, inhibe la actividad osteoblástica y estimula la osteoclástica. ${ }^{96,97}$ Una ingestión menor a 600 mg diarios de calcio se con- sidera una variable más implicada en la osteopenia en mujeres adolescentes. ${ }^{25}$ Sin embargo, algunos autores no han encontrado asociación entre la ingestión de calcio o vitamina $\mathrm{D}$ consumidos a partir de la dieta y la $\mathrm{DMO}, 29,37,51,98$ posiblemente porque el periodo de evaluación ha sido corto. ${ }^{99}$

\section{Factores de riesgo clínico en pacientes con AN}

La pérdida de DMO está relacionada negativamente con el peso, ${ }^{51,54}$ es decir, a menor peso alcanzado ${ }^{53,100,101}$ y mayor tiempo de permanencia en éste ${ }^{67}$ mayor pérdida de masa ósea se tendrá. La pérdida de masa magra es un factor pronóstico importante para la pérdida de masa ósea, ${ }^{102}$ con una correlación significativa entre ésta y la DMO lumbar ${ }^{103,104}$ y total. ${ }^{103}$ El peso es también un factor pronóstico de la recuperación, pues se ha visto que el incremento de peso por año es mayor si el IMC menor alcanzado es $>16.4 \mathrm{~kg} / \mathrm{m}^{2} .{ }^{67}$ Por su parte, aquellas pacientes que logran un IMC mayor a 19 cuando son dadas de alta en clínicas especializadas, tienen una DMO mayor unos años después que quienes no alcanzaron este peso. ${ }^{105}$

La duración de la enfermedad es un factor predictivo de DMO trabecular ${ }^{25,28,30,32,61,98}$ y la mejor variable para predecir DMO cortical. ${ }^{98}$ Doce meses después del inicio de la AN la DMO ya se encuentra disminuida. ${ }^{25,103}$

Algunos autores han encontrado que si la AN comienza antes de la menarca ${ }^{56,66}$ se tiene un peor pronóstico para la DMO lumbar en la edad adulta, mientras que otros ${ }^{72}$ han detectado que mujeres diagnosticadas con AN después de los 20 años de edad tienen mayor incidencia de fracturas, comparadas con aquellas diagnosticadas en edades más tempranas.

El ejercicio se considera un factor protector de DMO en pacientes con $\mathrm{AN} .^{25,26,32,38,54}$ Sin embargo, hecho de manera extenuante, tiene un efecto perjudicial. ${ }^{36,71}$ El ejercicio "contra-peso" puede compensar los efectos del hipogonadismo, predominantemente en zonas de hueso cortical que soportan carga. ${ }^{38,106} \mathrm{Algu}-$ nos autores ${ }^{29,98,100}$ no han encontrado correlación entre ejercicio y DMO quizá porque los periodos de evaluación han sido breves. ${ }^{107}$

\section{Tratamiento farmacológico para la osteopenia en la AN}

La ganancia de peso juega un papel crucial para la recuperación de la masa ósea $29,31,66,67,78,79,108$ y restablece el desacoplamiento del remodelado óseo; ${ }^{80}$ por lo tanto, la terapia nutricional es esencial ${ }^{79,99,109}$ y efectiva para in- 
crementar marcadores de formación ósea. ${ }^{88}$ El entendimiento de los procesos fisiopatológicos implicados en la pérdida de masa ósea en la $\mathrm{AN}$, ha dado pie a la implementación de tratamientos farmacológicos que busquen frenar o disminuir el déficit. $65,66,103,110,111$

La eficacia absoluta del tratamiento con remplazo hormonal en pacientes con AN no se ha demostrado. ${ }^{26,65,68,112,113} \mathrm{Su}$ efecto no ha sido suficiente para reducir la pérdida de masa ósea cuando se ha suplementado por periodos entre 9 y 18 meses, como se muestra en el cuadro I. La efectividad de los estrógenos para incrementar o preservar la DMO en mujeres con AN está mitigada por la desnutrición continua, ${ }^{51}$ pues la DMO en columna lumbar ${ }^{51,58}$ y cadera total ${ }^{51}$ en mujeres con AN que toman anticonceptivos orales, no es diferente de aquéllas que no los ingieren.

En el cuadro II se listan otras estrategias farmacológicas estudiadas para el tratamiento de la pérdida de masa ósea. El tratamiento combinado que llevó a cabo Grinspoon y su equipo, ${ }^{104}$ logró un incremento significativo de DMO lumbar en donde la terapia de remplazo hormonal potencializó el efecto anabólico del FCI-1.

Respecto de la suplementación con calcio, aunque existen evidencias de que $1000 \mathrm{mg}$ de calcio diarios en adolescentes sanas puede elevar la DMO en columna dentro de los primeros seis meses de tratamiento, ${ }^{114}$ en pacientes con AN no se ha observado beneficio. ${ }^{29,102}$

\section{Repercusión de la pérdida de masa ósea a largo plazo en la AN}

Existen pruebas de que la osteopenia persiste aún después de la recuperación del peso y la menstruación 27,68,115 pues la recuperación de la DMO es muy lenta, ${ }^{27,31,116}$ especialmente en hueso cortical. ${ }^{37,98,99}$ Después de casi 12 años de la primera admisión, ${ }^{98}$ y 25 años después de una recuperación completa, ${ }^{115}$ no se logran reponer las pérdidas.

Varios estudios han afirmado que la DMO se normaliza después de la recuperación, pero no han incluido una medición inicial con la cual se haya podido establecer alguna comparación. ${ }^{61,108,117}$ Las adolescentes jóvenes con un curso de enfermedad corto (17 meses), y que logran una recuperación adecuada del peso (IMC>19), tienen un incremento de la DMO tres veces mayor que la ganancia observada en las adolescentes sanas. Por ello, se piensa que este grupo pudiera tener mayores posibilidades de recuperación. ${ }^{64}$ Sin embargo, existe un incremento en el riesgo de fractura en las pacientes con AN después de un año del diagnóstico y por más de 10 años después de éste, ${ }^{72}$ siendo 2.9 veces

Cuadro I

ESTUdios QUE DEMUESTRAN LA INEFICACIA DE LA TERAPIA SUSTITUTIVA HORMONAL

\begin{tabular}{|c|c|c|c|c|c|}
\hline Autor & Diseño & $n$ & Edad rango & Intervención & Hallazgo \\
\hline $\begin{array}{l}\text { Kreipe }^{26} \\
1993\end{array}$ & Transversal & 4 & $17-28$ & Biopsias de hueso & $\begin{array}{l}\text { La terapia hormonal no incrementó la formación ósea } \\
\text { en las dos mujeres con AO*. }\end{array}$ \\
\hline $\begin{array}{l}\text { Klibansky } \\
1995\end{array}$ & $\begin{array}{l}\text { Experimental } \\
\text { aleatorio }\end{array}$ & 48 & $16-42$ & $\begin{array}{l}\text { Tratamiento con o sin estro- } \\
\text { progesterona por } 1.5 \text { años }\end{array}$ & $\begin{array}{l}\text { No se observó cambio significativo en DMO en las } \\
\text { mujeres con tratamiento hormonal. }\end{array}$ \\
\hline $\begin{array}{l}\text { De la Piedra }{ }^{58} \\
1999\end{array}$ & Transversal & 41 & $16-24$ & $\begin{array}{l}\text { Estudió metabolismo óseo y } \\
\text { DMO }\end{array}$ & $\begin{array}{l}\text { No hubo diferencia significativa de la DMO e indicadores } \\
\text { de remodelado óseo entre pacientes con y } \sin \mathrm{AO} \text {. }\end{array}$ \\
\hline $\begin{array}{l}\text { Grispoon }{ }^{104} \\
2002\end{array}$ & $\begin{array}{l}\text { Experimental } \\
\text { aleatorio }\end{array}$ & 60 & $18-38$ & $\begin{array}{l}4 \text { grupos: } \\
\mathrm{FCl}-I^{\ddagger} \\
\mathrm{FCl}-1+\mathrm{AO} \\
\mathrm{AO} \\
\mathrm{Nada} \\
\text { Por nueve meses }\end{array}$ & $\begin{array}{l}\text { No hubo diferencia significativa en DMO lumbar entre } \\
\text { el grupo que recibió únicamente } A O \text { y el grupo sin } \\
\text { tratamiento }\end{array}$ \\
\hline $\begin{array}{l}\text { Golden } 66 \\
2002\end{array}$ & $\begin{array}{l}\text { Experimental } \\
\text { no aleatorio }\end{array}$ & 43 & $|3-2|$ & $\begin{array}{l}\text { Tratamiento con o } \sin \mathrm{AO} \text { por un } \\
\text { año }\end{array}$ & $\begin{array}{l}\text { No hubo diferencia significativa en el cambio neto de } \\
\text { DMO entre ambos grupos. }\end{array}$ \\
\hline $\begin{array}{l}\text { Muñoz } \\
2002\end{array}$ & Transversal & 38 & $\begin{array}{c}\bar{X} \\
17.3\end{array}$ & $\begin{array}{l}\text { Observó el cambio de DMO } \\
\text { después de un año de terapia con } \\
\text { estrógenos }\end{array}$ & $\begin{array}{l}\text { No hubo incremento significativo de DMO después de } \\
\text { la recuperación parcial del peso y terapia con } \\
\text { estrógenos. }\end{array}$ \\
\hline
\end{tabular}




\section{Cuadro II}

Otras estrategias farmacológicas para el tratamiento de la pérdida de masa ósea

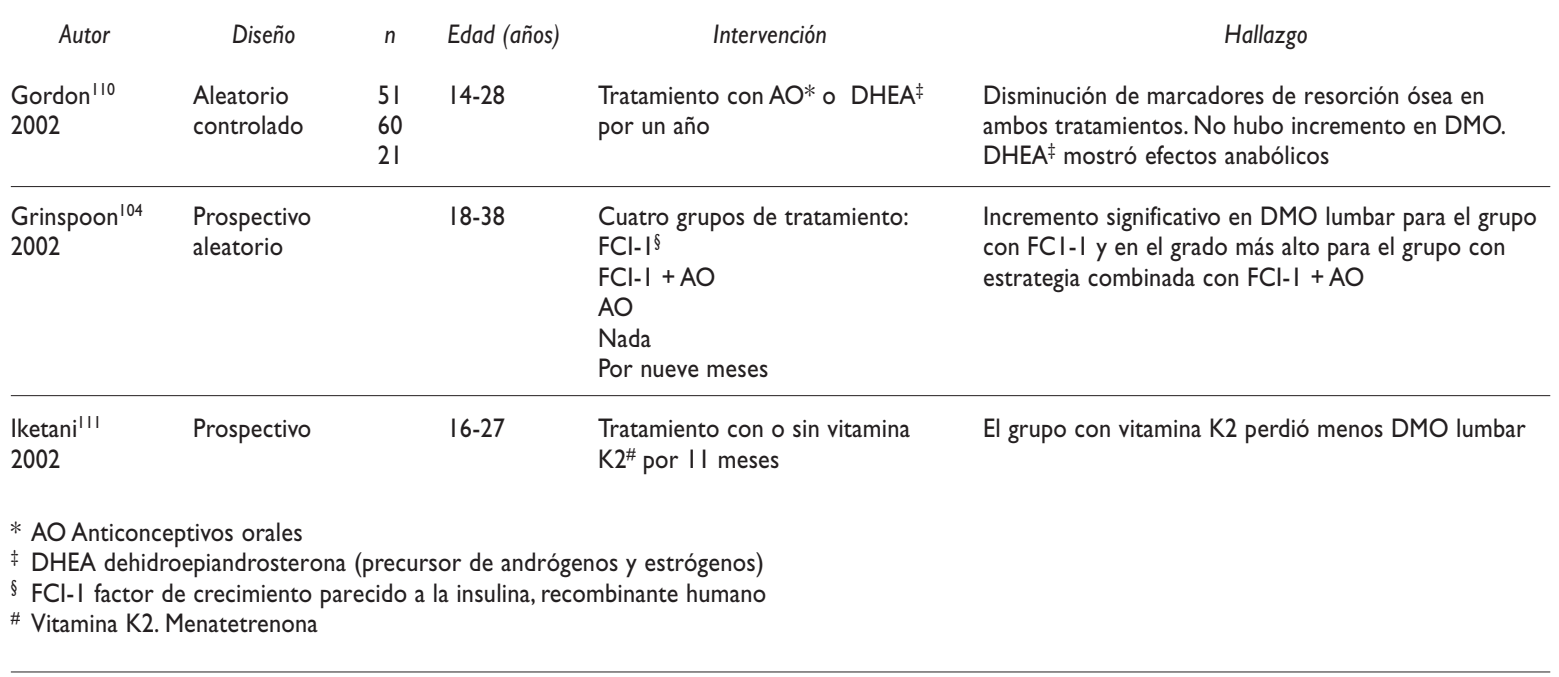

mayor en mujeres que han sufrido AN que en la población general. Esto se ha presentado con una frecuencia mayor en fractura de cadera, columna y radio distal, y ha ocurrido en promedio a los 38, 25 y 24 años después del diagnóstico, respectivamente, con una incidencia acumulativa de $57 \%$ para cualquier fractura, 40 años después del diagnóstico. ${ }^{102}$

\section{Recomendaciones para el tratamiento de la osteopenia en la AN}

A pesar de que no existen estudios que comprueben la eficacia de la suplementación con calcio y vitamina $D$, ésta se recomienda para quienes no cumplan con las ingestiones dietéticas de referencia de calcio, ${ }^{62,66}$ de entre 1000 y $1500 \mathrm{mg}$ diarios ${ }^{109,118,119}$ junto con 400 a 600 UI de vitamina $D^{62,66,109}$ y un régimen de ejercicio moderado ${ }^{49,54,62}$ del tipo "de carga de peso"62 junto con la recuperación del peso. ${ }^{49}$

La decisión de suplementar con estrógenos debe ser individualizada ${ }^{104} \mathrm{y}$, dado el riesgo potencial de fractura y el hecho de que la AN es frecuentemente refractaria al tratamiento, la terapia sustitutiva hormonal para las pacientes adultas o en la adolescencia tardía no debe ser completamente abandonada..$^{23,120}$ Una desventaja de este tratamiento es que va a enmascarar el momento cuando el cuerpo ha llegado a una recuperación biológica, dando un falso sentido de seguridad a las mujeres que aún se encuentran en un peso bajo y están teniendo ciclos menstruales regulares. ${ }^{65,119}$

La densitometría es un instrumento útil para identificar y advertir a las pacientes que se encuentran por debajo del valor esperado para su edad; asimismo, provee una oportunidad para hacer constar a la paciente sobre los efectos a largo plazo del peso bajo. ${ }^{63,109}$ Debe indicarse cuando se tiene un IMC $<15$ una ingestión de calcio menor a $600 \mathrm{mg}$ diarios, una duración del trastorno mayor a 12 meses o una duración de amenorrea mayor a seis meses, ${ }^{23,25,63}$ y repetirse cada dos años hasta la recuperación del peso y de ciclos menstruales regulares, ${ }^{23} \mathrm{O}$ cada año si hay un incremento pequeño y constante de peso. ${ }^{63}$

\section{Conclusiones}

La AN es el padecimiento más susceptible de presentar DMO disminuida. El TANE y la BN también son grupos vulnerables, el primero porque puede representar un periodo preclínico de la AN, y el segundo porque es común que se presente con antecedentes de AN. El efecto se hace evidente en hueso trabecular, antes que cortical.

El grado de deficiencia de masa ósea está relacionado con la severidad de la AN en función de su duración y el grado de pérdida de peso. No se tiene claro si la edad de inicio de la enfermedad, independientemente de su duración, tiene un efecto en la DMO. El sedentarismo se identificó como un factor de riesgo para deterioro del desarrollo de la masa ósea; se habla incluso de que el ejercicio "contra peso" neutraliza los efectos del hipogonadismo en zonas de hueso que soportan carga. Sin embargo, se requieren estudios que evalúen esta variable a largo plazo para determinar la frecuencia e intensidad adecuadas, y el momento opor- 
tuno dentro del proceso de recuperación en que debe iniciarse sin que éste interfiera con la ganancia de peso.

Los mecanismos implicados en la patogénesis de la deficiencia de DMO son el hipoestrogenismo, hipercorticismo, concentraciones bajas de leptina, triyodotironina y FCI-1. En cuanto a factores dietéticos como ingestión de calcio y vitamina D no se encontró correlación con la $\mathrm{DMO}$ porque los periodos de evaluación no fueron suficientemente largos. ${ }^{107} \mathrm{La}$ acidosis metabólica, consecuencia de la inanición, se supone también como factor etiológico importante.

La repercusión clínica del deterioro de la DMO en relación con el incremento en el riesgo de fractura en la vida futura es un dato claro que confirma a la AN como factor de riesgo para osteoporosis en la vida adulta, y hace evidente la necesidad de incluir en los cuestionarios que actualmente se realizan en campañas masivas de identificación de adultos en riesgo de padecer osteoporosis, el antecedente de TCA en sus años adolescentes y su perpetuación en la adultez.

En cuanto a la salud ósea, el tratamiento de la AN es complejo debido al curso crónico del trastorno y a la resistencia común que se presenta al cambio. Algunas de las alteraciones hormonales y endocrinas que derivan de la inanición, pérdida de peso, conducta purgativa o estrés intervienen en la perpetuación de los síntomas a través de la alteración del apetito, saciedad y estado de ánimo haciendo sumamente difícil la recuperación en términos de la realimentación y el incremento de peso, que es el factor principal para el restablecimiento del desacoplamiento en el remodelado óseo. Esto, aunado a la falta de estrategias farmacológicas adecuadas para contrarrestar el desarrollo insuficiente de la masa ósea, impide dar un tratamiento eficaz.

La efectividad de la terapia de remplazo hormonal para aumentar la DMO en mujeres con AN no se ha demostrado. La decisión de utilizarla en la edad adulta o adolescencia tardía debe ser individualizada. La terapia sustitutiva hormonal combinada con la suplementación de FCI-1 puede tener un efecto sinérgico, como lo comprobaron Grinspoon y su equipo. ${ }^{104} \mathrm{La}$ intervención con este método no es útil porque requiere de un monitoreo constante de la glicemia.

Para lograr la recuperación del estado de nutrición y del peso es necesaria la integración de la atención psicológica, psiquiátrica, médica y de nutrición. Al momento, sólo se han sugerido medidas generales para el tratamiento del desarrollo deficiente de masa ósea. Se recomienda la recuperación del peso corporal, ingestión adecuada de vitamina $\mathrm{D}$ y calcio, así como un régimen de ejercicio moderado del tipo "contra peso". Es necesario diseñar estrategias farmacológicas que a la par de estas medidas logren optimizar el desarrollo de la masa ósea para reponer las deficiencias que en su momento fueron ocasionadas por el TCA. Es importante que el equipo multidisciplinario preste atención especial a las pacientes anorécticas con una duración de la amenorrea mayor a seis meses, un IMC menor a $15 \mathrm{~kg} / \mathrm{m}^{2}$ o una ingestión de calcio menor a $600 \mathrm{mg}$ diarios. Cuando alguna de estas condiciones está presente el riesgo de presentar deficiencia en el desarrollo de la masa ósea es alto, por consiguiente se debe indicar un estudio densitométrico.

Por otra parte, el deterioro de la masa ósea que inducen los TCA en adolescentes o adultos jóvenes que aún no alcanzan el pico de masa ósea, es decir, la acumulación máxima de los componentes de la estructura de los huesos, representa el grado de ineficiencia para alcanzar dicha característica de las reservas del esqueleto. Los autores de las publicaciones consultadas utilizaron los términos osteoporosis y osteopenia por la popularidad de la clasificación de la OMS; sin embargo, no son aplicables para definir datos densitométricos de poblaciones jóvenes que no han alcanzado su reserva ósea pico. Más aún, esto es una limitación para ser capaces de proporcionar un enfoque claro al efecto de los TCA en la salud ósea en poblaciones jóvenes. En la actualidad, no existe consenso entre expertos ni una nomenclatura aceptada que le dé nombre propio a las deficiencias en el desarrollo de la reserva ósea antes de que ésta alcance su máxima concentración. Por ello, se considera relevante asignar un nombre a este trastorno diferente a la osteoporosis en la adultez. La palabra "hipo-osteogenesia" lo define de forma correcta, independientemente del padecimiento que lo ocasione. La raíz griega "genesia" significa producción, ${ }^{121}$ por lo tanto al añadir el prefijo "hipo" estaríamos refiriéndonos a una producción o desarrollo deficiente del tejido óseo. Asimismo, para definir la hipo-osteogenesia de muy mal pronóstico en la AN crónica, se agregaría el adjetivo "ominosa", del latín "ominosus" para denominarla como "hipo-osteogenesia ominosa de la AN".

La Encuesta Nacional de Epidemiología Psiquiátrica del Instituto Nacional de Psiquiatría Ramón de la Fuente Muñiz, cuyo blanco fue la población no institucionalizada mayor de 18 años, ya incluyó datos sobre TCA y detectó una prevalencia de bulimia nervosa de $6 \%$ en hombres y $1.8 \%$ en mujeres. ${ }^{12}$ Sin embargo, para tener una visión clara de la magnitud del daño que ocasionan y emprender programas preventivos, las futuras líneas de investigación en México deberán enfocarse, en primer término, en investigar la prevalencia e incidencia de los TCA en una muestra que incluya adolescentes. Además, deben hacerse estudios 
prospectivos a largo plazo que permitan aclarar los mecanismos implicados en la patogénesis de la "hipoosteogenesia" para diseñar estrategias de intervención adecuadas. Es indispensable evaluar el efecto de estrategias combinadas con estrógenos y métodos anabólicos en la recuperación de la masa ósea. El entendimiento de las alteraciones en algunos sistemas de neuropéptidos que intervienen en la conducta alimentaria ayudarán a aclarar su influencia en la resistencia al tratamiento, así como su posible participación en la etiología de la AN y BN. ${ }^{74}$ El análisis genético de la AN ayudará a identificar nuevas estrategias de medicación y tratamiento. ${ }^{89}$

\section{Agradecimientos}

Agradecemos al Dr. Edmundo Calva, investigador titular del Instituto de Biotecnología de la UNAM, por su apoyo para la recolección de diversos artículos.

\section{Referencias}

I.American Psychiatric Association. Diagnostic and statistical manual of mental disorder. Washington, DC:APA; 1994.

2. World Health Organization. The ICD-I0 classification of mental and behavioral disorder: Clinical description and diagnostic guidelines. Geneva:WHO; 1992.

3. Clinton D, Button E, Norring C, Palmer R. Cluster analysis of key diagnostic variables from two independent samples of eating-disorder patients: Evidence for a consistent pattern. Psychol Med 2004;34: 1035 1045.

4. Yager J,Andersen AE, Devlin M, Egger G, Herzog D, Mitchell J. III. Disease definition, epidemiology and natural history. Practice guidelines for the treatment of patients with eating disorders. Disponible en: http:/ /www.psych.org/clin_res/quide.bk4cfm.

5. Bulik CM, Sullivan PF, Fear J, Pickering A. Predictors of the development of bulimia nervosa in women with anorexia nervosa. J Nerv Ment Dis 1997;185:704-707.

6. Rojo L, Livianos L, Conesa L, García A, Domínguez A, Rodrigo G et al. Epidemiology and risk factors of eating disorders: $A$ two-stage epidemiologic study in a Spanish population aged I2-18 years. Int J Eat Disord 2003;34:28I-29I.

7. Hoek HW, van Hoeken D. Review of the prevalence and incidence of eating disorders. Int J Eat Disord 2003;34:383-396.

8. Pérez-Gaspar M, Gual P, De Irala-Estévez J, Martínez-González MA, Lahortiga F, Cervera S. Prevalencia de los transtornos de la conducta alimentaria en los adolescentes navarros. Med Clin 2000; | |4:48 I-486. 9. Lucas AR, Crowson CS, O'Fallon WM, Melton 3rd LJ. The ups and downs of anorexia nervosa. Int J Eat Disord 1999;26:397-405.

10. Nielsen S. Epidemiology and mortality of eating disorders. Psychiatr clin North Am 200I;24:210-214.

I I. Unikel SC, Villatoro VJA, Medina-Mora IME, Fleiz-Bautista C, AlcántarMolina EN, Hernández-Rosario SA. Conductas alimentarias de riesgo en adolescentes mexicanos. Datos en población estudiantil del Distrito Federal. Rev Invest Clin 2000;52: I40-I 47.

12. Medina-Mora IME, Borges G, Lara-Muñoz C, Benjet C, Blanco-Jaimes J, Fleiz-Bautista C. Prevalencia de trastornos mentales y uso de servicios. Resultados de la Encuesta Nacional de Epidemiología Psiquiátrica en México. Salud Mental 2003;26:1-16.

13. Herzog W, Deter HC, Fiehn W, Petzold E. Medical findings and predictors of long-term physical outcome in anorexia nervosa: $A$ prospective, I2-year follow-up study. Psychol Med 1997;27:269-279. 14.Asociación Mexicana de Metabolismo Oseo y Mineral. Consejo Mexicano de Osteoporosis. Rev Invest Clin 200I;53:469-495. 15. The World Health Organization Study Group. Assesment of fracture risk and its application to screening for postmenopausal osteoporosis. Technical report series No. 843. Ginebra:WHO; 1994.

16. Stini WA. Osteoporosis. Etiologies, prevention and treatment. Year Book Physic Anthropol 1990;33: I 5 I- 194.

17. Parra-Cabrera MS, Hernández-Avila M,Tamayo y Orozco JA, Fernández-Ortega MC, Meneses F. Factores de riesgo en la osteoporosis: evidencias clínicas y epidemiológicas. Gac Med Mex 1994;4:231-240.

18. Gordon CM. Bone density issues in the adolescent gynecology patient. J Pediatr Adolesc Gynecol 2000;13:157-161.

19. Riggs BL, Melton 3rd LJ. Ilnvolutional osteoporosis. N Engl J Med 1986;314:1676-1686.

20. Key JD, Key Jr LL. Calcium needs of adolescents. Curr Opin Pediatr 1994;6:379-382.

21. Bachrach LK. Bone mineralization in childhood and adolescence. Curr Opin Pediatr 1993;5:467-473.

22. Sharp CW, Freeman CP.The medical complications of anorexia nervosa. Br J Psychiatry 1993;162:452-462.

23. Mehler PS, Krantz M. Anorexia nervosa medical issues. J Womens Health 2003;12:331-340.

24. Savvas M, Treasure J, Studd J, Fogelman I, Moniz C, Brincat M. The effect of anorexia nervosa on skin thickness, skin collagen and bone density. Br J Obstet Gynaecol 1989;96:1392-1394.

25. Castro J, Lázaro L, Pons F, Halperin I, Toro J. Predictors of bone mineral density reduction in adolescents with anorexia nervosa. J Am Acad Child Adolesc Psychiatry 2000;39:1365-1370.

26. Seeman E, Szmukler GI, Formica C,Tsalamandris C, Mestrovic R. Osteoporosis in anorexia nervosa:The influence of peak bone density, bone loss, oral contraceptive use, and exercise. J Bone Miner Res 1992;7:1467-1474.

27. Ward A, Brown N, Treasure J. Persistent osteopenia after recovery from anorexia nervosa. Int J Eat Disord 1997;22:7I-75.

28. Biller BM, Saxe V, Herzog DB, Rosenthal DI, Holzman S, Klibanski A. Mechanisms of osteoporosis in adult and adolescent women with anorexia nervosa.J Clin Endocrinol Metab 1989;68:548-554. 29. Bachrach LK, Guido D, Katzman D, Litt IF, Marcus R. Decreased bone density in adolescent girls with anorexia nervosa. Pediatrics 1990;86:440-447.

30. Poet JL, Galinier PA, Tonolli SI, Conte DB, Roux H. Lumbar bone mineral density in anorexia nervosa. Clin Rheumatol 1993; 12:236-239. 3I. Zipfel S, Seibel MJ, Lowe B, Beumont PJ, Kasperk C, Herzog W. Osteoporosis in eating disorders: A follow-up study of patients with anorexia and bulimia nervosa. J Clin Endocrinol Metab 200I;86:52275233.

32. Andersen AE, Woodward PJ, LaFrance N. Bone mineral density of eating disorder subgroups. Int J Eat Disord 1995; | 8:335-342. 33. Newman MM, Halmi KA. Relationship of bone density to estradiol and cortisol in anorexia nervosa and bulimia. Psychiatry Res 1989;29:105-112.

34. Kaplan FS, Pertschuk M, Fallon M, Haddad J. Osteoporosis and hip fracture in a young woman with anorexia nervosa. Clin Orthop Relat Res 1986;212:250-254.

35. Siemers B, Chakmakjian Z, Gench B. Bone density patterns in women with anorexia nervosa. Int J Eat Disord 1996;19:179-186. 36. Joyce JM, Warren DL, Humphries LL, Smith AJ, Coon JS. Osteoporosis in women with eating disorders: Comparison of physical 
parameters, exercise, and menstrual status with SPA and DPA evaluation. J Nucl Med 1990;31:325-331.

37. Rigotti NA, Neer RM, Skates SJ, Herzog DB, Nussbaum SR. The clinical course of osteoporosis in anorexia nervosa. A longitudinal study of cortical bone mass. JAMA 1991;265: | |33-1 |38.

38. Rigotti NA, Nussbaum SR, Herzog DB, Neer RM. Osteoporosis in women with anorexia nervosa. N Engl J Med 1984;3 II:I60 I-I606. 39. Brotman AW, Stern TA. Osteoporosis and pathologic fractures in anorexia nervosa. Am J Psychiatry 1985; I42:495-496.

40. Gillberg C, Rastam M, Gillberg IC. Anorexia nervosa: Physical health and neurodevelopment at 16 and 21 years. Dev Med Child Neurol 1994;36:567-575.

4I. Baum ML, Kramer EL, Sanger J], Peña A. Stress fractures and reduced bone mineral density with prior anorexia nervosa.J Nucl Med 1987;28:1506-1507.

42. Warren MP, Shane E, Lee MJ, Lyndsay R, Dempster DW, Warren LF et al. Femoral head collapse associated with anorexia nervosa in a 20-yearold ballet dancer. Clin Orthop Relat Res 1990;25I:I7I-I76.

43. McAnarney ER, Greydanus DE, Campanella VA, Hoekelman RA. Rib fractures and anorexia nervosa. J Adolesc Health Care 1983;4:40-43. 44. Root AW, Powers PS. Anorexia nervosa presenting as growth retardation in adolescents. J Adolesc Health Care 1983;4:25-30. 45. Danziger Y, Mukamel M, Zeharia A, Dinari G, Mimouni M. Stunting of growth in anorexia nervosa during the prepubertal and pubertal period. Isr J Med Sci 1994;80:581-584.

46. Pugliese MT, Lifshitz F, Grad G, Fort P, Marks-Katz M. Fear of obesity. A cause of short stature and delayed puberty. $N$ Engl J Med 1983;309:513-518.

47. Modan-Moses D, Yaroslavsky A, Novikov I, Segev S, Toledano A, Miterany $E$ et al. Stunting of growth as a major feature of anorexia nervosa in male adolescents. Pediatrics 2003; | | 1:270-276. 48. Soyka LA, Grinspoon S, Levitsky LL, Herzog DB, Klibanski A.The effects of anorexia nervosa on bone metabolism in female adolescent. J Clin Endocrinol Metab 1999;84:4489-4496.

49. Muñoz MT,Argente J.Anorexia nervosa: Hypogonadotrophic hypogonadism and bone mineral density. Horm Res 2002;57:S57-S62. 50. Golden NH, Kreitzer P, Jacobson MS, Chasalow Fl, Schebendach J, Freedman SM et al. Disturbances in growth hormone secretion and action in adolescents with anorexia nervosa.J Pediatr 1994; 125:655-660. 5I. Grinspoon S, Thomas E, Pitts S, Gross E, Mickley D, Miller K et al. Prevalence and predictive factors for regional osteopenia in women with anorexia nervosa. Ann Intern Med 2000;133:790-794.

52. Resch H, Newrkla S, Grampp S, Resch A, Zapf S, Piringer S et al. Ultrasound and $\mathrm{X}$-ray-based bone densitometry in patients with anorexia nervosa. Calcif Tissue Int 2000;66:338-34I.

53. Lennkh C, De Zwaan M, Bailer U, Strnad A, Nagy C, El-Giamal N et al. Osteopenia in anorexia nervosa: Specific mechanisms of bone loss. J Psychiatr Res 1999;33:349-356.

54. Gordon CM, Goodman E, Emans SJ, Grace E, Becker KA, Rosen JC et al. Physiologic regulators of bone turnover in young women with anorexia nervosa. J Pediatr 2002; 14:64-70.

55. Calero JA, Muñoz MT, Argente J,Traba ML, Méndez-Dávila C, GarcíaMoreno $C$ et al. A variation in Bone Alkaline Phosphatase levels that correlates positively with bone loss and normal levels of aminoterminal propeptide of collagen I in girls with anorexia nervosa. Clin Chim Acta 1999;285: 121-129.

56. Salisbury JJ, Mitchell JE. Bone mineral density and anorexia nervosa in women. Am J Psychiatry 199|;|48:768-774.

57. Grinspoon S, Baum H, Lee K,Anderson E, Herzog D, Klibanski A. Effects of short-term recombinant human insulin-like growth factor I administration on bone turnover in osteopenic women with anorexia nervosa. J Clin Endocrinol Metab 1996;81:3864-3870.

58. De la Piedra C, Calero JA, Traba ML, Asensio MD,Argente J, Muñoz MT. Urinary alpha and beta C-telopeptides of collagen I: clinical implications in bone remodeling in patients with anorexia nervosa. Osteoporos Int 1999;10:480-486.

59. Castro J, Toro J, Lázaro L, Pons F, Halperin I. Bone mineral density in male adolescents with anorexia nervosa. J Am Acad Child Adolesc Psychiatry 2002;41:613-618.

60. Andersen AE, Watson T, Schlechte J. Osteoporosis and osteopenia in men with eating disorders. Lancet 2000;355:7967-1968. 6I. Wentz E, Mellstrom D, Gillberg C, Sundh V, Gillberg IC, Rastam M. Bone density II years after anorexia nervosa onset in a controlled study of 39 cases. Int J Eat Disord 2003;4:314-318.

62. Golden NH. Osteopenia and osteoporosis in anorexia nervosa. Adolesc Med 2003; 14:97-108.

63. Rome ES. Eating disorders. Obstet Gynecol Clin North Am 2003;30:353-377.

64. Castro J, Lázaro L, Pons F, Halperin I, Toro J.Adolescent anorexia nervosa:The catch-up effect in bone mineral density after recovery.J Am Acad Child Adolesc Psychiatry 2001;40:1215-1221.

65. Maugars YM, Berthelot JM, Forestier R, Mammar N, Lalande S, Venisse JL et al. Follow-up of bone mineral density in 27 cases of anorexia nervosa. Eur J Endocrinol 1996; 135:59|-597.

66. Golden NH, Lanzkowsky L, Schebendach J, Palestro CJ, Jacobson MS, Shenker IR. The effect of estrogen-progestin treatment on bone mineral density in anorexia nervosa.J Pediatr Adolesc Gynecol 2002; 15: I35-I43. 67. Hotta M, Shibasaki T, Sato K, Demura H. The importance of body weight history in the occurrence and recovery of osteoporosis in patients with anorexia nervosa: Evaluation by dual X-ray absorptiometry and bone metabolic markers. Eur J Endocrinol 1998; 139:276-283.

68. Karlsson MK, Weigall SJ, Duan Y, Seeman E. Bone size and volumetric density in women with anorexia nervosa receiving estrogen replacement therapy and in women recovered from anorexia nervosa. J Clin Endocrinol Metab 2000;85:3177-3182.

69. Sundgot-Borgen J, Bahr R, Falch JA, Schneider LS. Normal bone mass in bulimic women.J Clin Endocrinol Metab 1998;83:3।44-3।49.

70. Newton JR, Freeman CP, Hannan WJ, Cowen S. Osteoporosis and normal weight bulimia nervosa--which patients are at risk? J Psychosom Res 1993;37:239-247.

7I. Goebel G, Schweiger U, Kruger R, Fichter MM. Predictors of bone mineral density in patients with eating disorders. Int J Eat Disord 1999;25:143-150.

72. Vestergaard P, Emborg C, Stoving RK, Hagen C, Mosekilde L, Brixen $\mathrm{K}$. Fractures in patients with anorexia nervosa, bulimia nervosa, and other eating disorders--a nationwide register study. Int J Eat Disord 2002;32:301-308.

73. Halmi K. Physiology of anorexia nervosa and bulimia nervosa. En: Fairburn CG, Brownell KD, Ed. Eating disorders and obesity. Nueva York: The Gilford Press; 2002:267-27I.

74. Kaye WH. Central nervous system neurotransmitter activity in anorexia nervosa and bulimia nervosa. En: Fairburn CG, Brownell KD, Ed. Eating disorders and obesity. Nueva York:The Gilford Press; 2002:272-277.

75. Grinspoon S, Miller K, Coyle C, Krempin J,Armstrong C, Pitts S et al. Severity of osteopenia in estrogen-deficient women with anorexia nervosa and hypothalamic amenorrhea. J Clin Endocrinol Metab 1999;84:2049-2055.

76. Miller K, Grinspoon S, Grieco KA, Ciampa J, Karen K. Miller MD SKGM, Neuroendocrine, Kelly A. Grieco BA, Julia Ciampa BA. Preservation of menstrual function in women who full fill criteria for anorexia nervosa. International Conference of Eating Disorders, April 29-May 2, 2004. Disponible en: http://www.hedc.org. 77. Gordon CM, Grace E, Emans SJ, Goodman E, Crawford MH, Leboff MS. Changes in bone turnover markers and menstrual function after short-term oral DHEA in young women with anorexia nervosa.J Bone Miner Res 1999;14:136-145. 
78. Kreipe RE, Hicks DG, Rosier RN, Puzas JE. Preliminary findings on the effects of sex hormones on bone metabolism in anorexia nervosa. J Adolesc Health 1993; 14:319-324.

79. Heer M, Mika C, Grzella I, Drummer C, Herpertz-Dahlmann B. Changes in bone turnover in patients with anorexia nervosa during eleven weeks of inpatient dietary treatment. Clin Chem 2002;48:754760 .

80. Caillot-Augusseau A, Lafage-Proust MH, Margaillan P,Vergely N, Faure $S$, Paillet $S$ et al. Weight gain reverses bone turnover and restores circadian variation of bone resorption in anorexic patients. Clin Endocrinol 2000;52:II3-121.

81. Heaney RP. Pathophysiology of osteoporosis. Endocrinol Metab Clin North Am 1998;27:255-265.

82. Caregaro L, Favaro A, Santonastaso P,Alberino F, Di Pascoli L, Nardi $M$ et al. Insulin-like growth factor I (IGF-I), a nutritional marker in patients with eating disorders. Clin Nutr 200I;20:25I-257. 83. Vergely N, Lafage-Proust MH, Caillot-Augusseau A, Millot L, Lang F, Estour B. Hypercorticism blunts circadian variations of osteocalcin regardless of nutritional status. Bone 2002;30:428-435.

84. Argente J, Caballo N, Barrios V, Muñoz MT, Pozo J, Chowen JA et al Multiple endocrine abnormalities of the growth hormone and insulinlike growth factor axis in patients with anorexia nervosa: Effect of short- and long-term weight recuperation. J Clin Endocrinol Metab 1997;82:2084-2092.

85. Counts DR, Gwirtsman H, Carlsson LM, Lesem M, Cutler Jr GB. The effect of anorexia nervosa and refeeding on growth hormone-binding protein, the insulin-like growth factors (IGFs), and the IGF-binding proteins. J Clin Endocrinol Metab 1992;75:762-767.

86. Klibanski A, Biller BM, Schoenfeld DA, Herzog DB, Saxe VC. The effects of estrogen administration on trabecular bone loss in young women with anorexia nervosa. J Clin Endocrinol Metab 1995;80:898904.

87. Hotta M, Fukuda I, Sato K, Hizuka N, Shibasaki T, Takano K. The relationship between bone turnover and body weight, serum insulin-like growth factor (IGF) I, and serum IGF-binding protein levels in patients with anorexia nervosa. J Clin Endocrinol Metab 2000;85:200-206.

88. Heer M, Mika C, Grzella I, Heussen N, Herpertz-Dahlmann B. Bone turnover during inpatient nutritional therapy and outpatient follow-up in patients with anorexia nervosa compared with that in healthy control subjects. Am J Clin Nutr 2004;80:774-78I.

89. Muñoz MT,Argente J.Anorexia nervosa in female adolescents: endocrine and bone mineral density disturbances. Eur J Endocrinol 2002;147:275-286

90. Muñoz MT, Morande G, García-Centenera JA, Hervas F, Pozo J, Argente J.The effects of estrogen administration on bone mineral density in adolescents with anorexia nervosa. Eur J Endocrinol 2002; | $46: 45-50$

91. Grinspoon S, Gulick T,Askari H, Landt M, Lee K, Anderson E et al. Serum leptin levels in women with anorexia nervosa.J Clin Endocrinol Metab 1996;81:3861-3863

92. Taylor AE, Hubbard J,Anderson El. Impact of binge eating on metabolic and leptin dynamics in normal young women. J Clin Endocrinol Metab 1999:84:428-434.

93. Herpertz S, Albers N, Wagner R, Pelz B, Kopp W, Mann K et al. Longitudinal changes of circadian leptin, insulin and cortisol plasma levels and their correlation during refeeding in patients with anorexia nervosa. Eur J Endocrinol 2000; 142:373-379.

94. Boyar RM, Hellman LD, Roffwarg H, et al. Cortisol secretion and metabolism in anorexia nervosa. N Engl J Med 1977;296:190-193. 95. Grinspoon S, Thomas L, Miller K, Pitts S, Herzog D, Klibanski A Changes in regional fat redistribution and the effects of estrogen during spontaneous weight gain in women with anorexia nervosa. Am J Clin Nutr 2001;73:865-869.
96. Bruni V, Dei M,Vicini I, Beninato L, Magnani L. Estrogen replacement therapy in the management of osteopenia related to eating disorders. Ann NY Acad Sci 2000;900:416-42I.

97. Krieger NS, Sessler NE, Bushinsky DA. Acidosis inhibits osteoblastic and stimulates osteoclastic activity in vitro. Am J Physiol 1992;262:442448.

98. Herzog W, Minne H, Deter C, Leidig G, Schellberg D, Wuster C et al. Outcome of bone mineral density in anorexia nervosa patients 1 I.7 years after first admission.J Bone Miner Res 1993;8:597-605. 99. Treasure J, Serpell L. Osteoporosis in anorexia nervosa. Hosp Med 1999:60:477-480.

100. Baker D, Roberts R, Towell T. Factors predictive of bone mineral density in eating-disordered women: a longitudinal study. Int J Eat Disord 2000;27:29-35.

10I.Wong S, Au B, Lau E, Lee Y, Sham A, Lee S. Osteoporosis in Chinese patients with anorexia nervosa. Int J Eat Disord 2004;36: I04-108. 102. Lucas AR, Melton 3rd LJ, Crowson CS, O'Fallon WM. Long-term fracture risk among women with anorexia nervosa:A population-based cohort study. Mayo Clin Proc 1999;74:972-977.

103. Wong JC, Lewindon P, Mortimer R, Shepherd R. Bone mineral density in adolescent females with recently diagnosed anorexia nervosa. Int J Eat Disord 2001;29:1 I-16.

104. Grinspoon S, Thomas L, Miller K, Herzog D, Klibanski A. Effects of recombinant human IGF-I and oral contraceptive administration on bone density in anorexia nervosa. J Clin Endocrinol Metab 2002;87:2883-2891.

105. Gross G, Russell JD, Beumont PJ, et al. Longitudinal study of patients with anorexia nervosa 6 to 10 years after treatment. Impact of adequate weight restoration on outcome.Ann NY Acad Sci 2000;904:6|4-6|6.

106. Young N, Formica C, Szmukler G, Seeman E. Bone density at weight-bearing and nonweight-bearing sites in ballet dancers:The effects of exercise, hypogonadism, and body weight. J Clin Endocrinol Metab 1994;78:449-454

107. Treasure J, Serpell L. Osteoporosis in young people. Research and treatment in eating disorders. Psychiatr Clin North Am 200 I;24:359370 .

108. Bachrach LK, Katzman DK, Litt IF, Guido D, Marcus R. Recovery from osteopenia in adolescent girls with anorexia nervosa. J Clin Endocrinol Metab 1991;72:602-606.

109. Grinspoon SK, Thomas ER. Bone loss in anorexia nervosa: mechanisms and treatment options. Disponible en: http:// www.gurze.net/site 12_5_00/boneloss.htm.

II0. Gordon CM, Grace E, Emans SJ, Feldman HA, Goodman E, Becher $\mathrm{KA}$ et al. Effects of oral dehydroepiandrosterone on bone density in young women with anorexia nervosa: A randomized trial.J Clin Endocrinol Metab 2002;87:4935-494I.

I I I. Iketani T, Kiriike N, Murray BS, Nagao K, Nagata T, Minamikawa N et al. Effect of menatetrenone (vitamin K2) treatment on bone loss in patients with anorexia nervosa. Psychiatry Res 2003; I 17:259-269. I 12. Powers PS. Osteoporosis and eating disorders. J Pediatr Adolesc Gynecol 1999;12:51-57.

I I3. Katzman DK. In opposition of HRT.J Pediatr Adolesc Gynecol 200I;| 14:39-4I

I I4. Nowson CA, Green RM, Hopper JL Sherwin AJ, Young D, Kaymakvi $B$ et al. A co-twin study of the effect of calcium supplementation on bone density during adolescence. Osteoporos Int 1997;7:219-225. I I5. Hartman D, Crisp A, Rooney B, Rackow C, Atkinson R, Patel S. Bone density of women who have recovered from anorexia nervosa. Int J Eat Disord 2000;28: 107-II2.

I 16. Seidenfeld ME, Rickert VI. Impact of anorexia, bulimia and obesity on the gynecologic health of adolescents. Am Fam Physician 2001;64:445-450 
I 17. Treasure JL, Russell GF, Fogelman I, Murby B. Reversible bone loss in anorexia nervosa. Br Med J 1987;295:474-475.

I 18. Standing Committee on the Scientific Evaluation of Dietary

Reference intakes food and nutrition board. Dietary reference intakes

for calcium, phosphorus, magnesium vitamin D and fluoride. Washington, DC: National Academy Press; 1997.

1 19. American Academy of Pediatrics Committee on Nutrition. Calcium requeriments of infants, children and adolescents. Pediatrics 1997;104:1152-1157.
120. Cromer B. In support of HRT.J Pediatr Adolesc Gynecol 200I;| 14:42-45.

I2I. Díaz Rojo JA. Nociones de neología. Las raíces griegas -génesis, genesia y -genia en la terminología médica. Consejo superior de investigaciones científicas. Disponible en: http://www.medtrad.org/ panacea/Indicegeneral 